\title{
Existence of Positive Solutions of Fisher-KPP Equations in Locally Spatially Variational Habitat with Hybrid Dispersal
}

\author{
Liang Kong ${ }^{1}$ \\ ${ }^{1}$ Department of Mathematical Sciences, University of Illinois at Springfield, Springfield, Illinois \\ Correspondence: Liang Kong, Department of Mathematical Sciences, University of Illinois at Springfield, Springfield, \\ Illinois, United States. Tel: 1-217-206-7219. E-mail: lkong9@uis.edu
}

Received: October 3, 2016 Accepted: December 5, 2016 Online Published: December 30, 2016

doi:10.5539/jmr.v9n1p1 URL: http://dx.doi.org/10.5539/jmr.v9n1p1

\begin{abstract}
The current paper investigate the persistence of positive solutions of KPP type evolution equations with random/nonlocal dispersal in locally spatially inhomogeneous habitat. By the constructions of super/sub solutions and comparison principle, we prove that such an equation has a unique globally stable positive stationary solution.
\end{abstract}

Keywords: KPP equations, random dispersal, nonlocal dispersal, localized spatial inhomogeneity, positive stationary solution, principal eigenvalue, sub-solution, super-solution, comparison principle

Mathematics subject classification. 35K57, 45G10, 58D20, 92D25.

\section{Introduction}

The current paper is concerned with persistence of species in locally spatially variational environments or habitat,

$$
u_{t}(t, x)=\mathcal{A} u(t, x)+u(t, x) f(x, u(t, x)), \quad x \in \mathbb{R}^{N}
$$

where $(\mathcal{A} u)(t, x)=v_{1} \Delta u+v_{2}\left[\int_{\mathbb{R}^{N}} \kappa(y-x) u(t, y) d y-u(t, x)\right], v_{1}, v_{2} \geq 0$ and $v_{1}+v_{2}>0$.

If $v_{2}=0$, then (1.1) is the classical reaction diffusion equation, so called random dispersal equation, (without loss of generality, let $v_{1}=1$ )

$$
u_{t}(t, x)=\Delta u(t, x)+u(t, x) f(x, u(t, x)), \quad x \in \mathbb{R}^{N}
$$

which is broadly used to model the population dynamics of many species in unbounded environments, where $u(t, x)$ is the population density of the species at time $t$ and location $x, \Delta u$ characterizes the internal interaction of the organisms, and $f(x, u)$ represents the growth rate of the population, which satisfies that $f(x, u)<0$ for $u \gg 1$ and $\partial_{u} f(x, u)<0$ for $u \geq 0$ (see Aronson \& Weinberger, 1957; Aronson \& Weinberger, 1978; Cantrell \& Cosner, 2003; Fife, 1979; Fife \& Peletier, 1977; Fisher, 1937; Kolmogorov, Petrowsky, \& Poscunov, 1937; Murray, 1989; Shigesada \& Kawasaki, 1997; Skellam, 1951; Weinberger, 1982; Weinberger, 2002; Zhao, 2003; etc.).

If $v_{1}=0$, then (1.1) is so called nonlocal dispersal equation, (without loss of generality, let $v_{2}=1$ )

$$
u_{t}(t, x)=\int_{\mathbb{R}^{N}} \kappa(y-x) u(t, y) d y-u(t, x)+u(t, x) f(x, u(t, x)), \quad x \in \mathbb{R}^{N},
$$

where $\kappa(\cdot)$ is a smooth convolution kernel supported on a ball centered at the origin (that is, there is a $\delta_{0}>0$ such that $\kappa(z)>0$ if $\|z\|<\delta_{0}, \kappa(z)=0$ if $\|z\| \geq \delta_{0}$, where $\|\cdot\|$ denotes the norm in $\mathbb{R}^{N}$ and $\delta_{0}$ represents the nonlocal dispersal distance), $\int_{\mathbb{R}^{N}} \kappa(z) d z=1$, and $f(\cdot, \cdot)$ is of the same property as $f$ in (1.2) (see Bates \& Zhao, 2007; Chasseigne, Chaves, \& Rossi, 2006; Cortazar, Coville, Elgueta, \& Martinez, 2007; Cousens, Dytham, \& Law, 2008; Fife, 2003; Grinfeld, Hines, Hutson, Mischaikow, \& Vickers, 2005; Hutson, Martinez, Mischaikow \& Vickers, 2003; Lee, Hoopes, Diehl, Gilliland, Huxel, Leaver, McCain, Umbanhowar, \& Mogilner, 2001; Levin, Muller-Landau, Nathan, \& Chave, 2003; etc.).

When using (1.2) to model the population dynamics of a species, it is assumed that the underlying environment is continuous and the dispersal of cells or organisms are based on the hypothesis that the movement of the dispersing species can be described as a random walk in which there is no correlation between steps. However, dispersal of large organisms often involves mechanisms that may introduce correlations in movements. To model the population dynamics of such species in the case that the underlying environment is continuous, the nonlocal dispersal equation (1.3) is often used. This paper propose to study a mixed dispersal strategy, that is, a hybrid of random and non-local dispersal. We assume that a fraction of individuals in the population adopt random dispersal, while the rest fraction assumes non-local dispersal. Some research has been done on the hybrid dispersal in the spatially periodic habitat (see Kao, Lou \& Shen, 2010; Kao, Lou \& Shen, 2012; and Zhang, 2013). Our main goal is to study how the hybrid dispersal affects the persistence of a 
single species and how the hybrid dispersal strategies will evolve in spatially locally inhomogeneous environment (see H1 and $\mathrm{H} 2$ ).

Since the seminal works by Fisher (Fisher, 1937) and Kolmogorov, Petrowsky, Piscunov (Kolmogorov, Petrowsky, \& Poscunov, 1937) on the following special case of (1.2),

$$
\frac{\partial}{\partial t} u(t, x)=\frac{\partial^{2}}{\partial x^{2}} u(t, x)+u(t, x)(1-u(t, x)), \quad x \in \mathbb{R} .
$$

A great deal of research has been carried out toward the spatial spreading dynamics of (1.2) and (1.3) with $f(\cdot, \cdot)$ being independent of the space variable or periodic in the space variable, which reflects the spatial periodicity of the media. We refer to (Aronson \& Weinberger, 1957; Aronson \& Weinberger, 1978; Berestycki, Hamel, \& Nadirashvili, 2010; Kametaka, 1976; Liang \& Zhao, 2007; Liang, Yi, \& Zhao, 2006; Sattinger, 1976; Uchiyama, 1978; Weinberger, 1982; etc). for the study of (1.2) in the case that $f(x, u)$ is independent of $x$ and refer to (Berestycki, Hamel, \& Nadirashvili, 2005; Berestycki, Hamel, \& Roques, 2005; Freidlin \& Gärtner, 1979; Hamel, 2008; Hudson \& Zinner, 1995; Nadin, 2009; Nolen, Rudd, \& Xin, 2005; Nolen \& Xin, 2005; Weinberger, 2002; etc). for the study of (1.2) in the case that $f(x, u)$ is periodic in $x$; refer to (Coville \& Dupaigne, 2005; Coville, Dávila, \& Martínez, 2008; Li, Sun, \& Wang, 2010; etc). for the study of (1.3) in the case that $f(x, u)$ is independent of $x$ and refer to (Hetzer, Shen, \& Zhang, 2013; Shen \& Zhang, 2012; etc.) for the study of (1.3) in the case that $f(x, u)$ is periodic in $x$ and refer to (Berestycki \& Nadin, 2016; Berestycki, Jin, \& Silvestre, 2016; Kong \& Shen, 2011; Kong \& Shen, 2014; Nolen, Roquejoffre, \& Ryzhik; Shen, 2011; etc). for the study of (1.2) and/or (1.3) in the case that $f(t, x, u)$ is temporally and/or spatially heterogeneous.

For example, consider (1.2) and assume that $f\left(x+p_{i} \mathbf{e}_{\mathbf{i}}, u\right)=f(x, u)$ for $i=1,2, \cdots, N$, where $p_{i}(i=1,2, \cdots, N)$ are positive constants and

$$
\mathbf{e}_{\mathbf{i}}=\left(\delta_{i 1}, \delta_{i 2}, \cdots, \delta_{i N}\right), \delta_{i j}=1 \text { if } i=j \text { and } 0 \text { if } i \neq j .
$$

If the principal eigenvalue of the following eigenvalue problem associated to the linearized equation of (1.2) at $u=0$,

$$
\left\{\begin{array}{l}
\Delta u(x)+f(x, 0) u(x)=\lambda u(x), \quad x \in \mathbb{R}^{N} \\
u\left(x+p_{i} \mathbf{e}_{\mathbf{i}}\right)=u(x), \quad x \in \mathbb{R}^{N},
\end{array}\right.
$$

is positive, then (1.2) has a unique positive stationary solution $u^{*}(\cdot)$ with $u^{*}\left(\cdot+p_{i} \mathbf{e}_{\mathbf{i}}\right)=u^{*}(\cdot)$.

In this paper, we consider (1.1) in the case that the growth rates depend on the space variable, but only when it is in some bounded subset of the underlying habitat, which reflects the localized spatial inhomogeneity of the media. More precisely, let $\mathcal{H}=\mathbb{R}^{N}$, we assume

(H1) $f: \mathcal{H} \times \mathbb{R} \rightarrow \mathbb{R}$ is a $C^{2}$ function, $f(x, u)<0$ for all $(x, u) \in \mathcal{H} \times \mathbb{R}^{+}$with $u \geq \beta_{0}$ for some $\beta_{0}>0$, and $\partial_{u} f(x, u)<0$ for all $(x, u) \in \mathcal{H} \times \mathbb{R}^{+}$.

(H2) $f(x, u)=f^{0}(u)$ for some $C^{2}$ function $f^{0}: \mathbb{R} \rightarrow \mathbb{R}$ and all $(x, u) \in \mathcal{H} \times \mathbb{R}$ with $\|x\| \geq L_{0}$ for some $L_{0}>0$, and $f^{0}(0)>0$.

Assume (H1) and (H2). Then (1.1) has the following limit equations as $\|x\| \rightarrow \infty$,

$$
u_{t}(t, x)=\mathcal{A} u(t, x)+u(t, x) f^{0}(u(t, x)), \quad x \in \mathbb{R}^{N} .
$$

Equations (1.6) will play an important role in the study of (1.1). Equations (1.6) has a unique positive constant stationary solution $u^{0}$. We introduce some standing notations and then state the main results of the paper.

Let $p=\left(p_{1}, p_{2}, \cdots, p_{N}\right)$ with $p_{i}>0$ for $i=1,2, \cdots, N$. We define the Banach spaces $X_{p}$ by

$$
X_{p}=\left\{u \in C\left(\mathbb{R}^{N}, \mathbb{R}\right) \mid u\left(\cdot+p_{i} \mathbf{e}_{\mathbf{i}}\right)=u(\cdot), \quad i=1, \ldots, N\right\}
$$

with norm $\|u\|_{X_{p}}=\max _{x \in \mathbb{R}^{N}}|u(x)|$.

Let

and

$$
X_{p}^{+}=\left\{u \in X_{p} \mid u(x) \geq 0 \forall x \in \mathcal{H}\right\}
$$

$$
X_{p}^{++}=\left\{u \in X_{p} \mid u(x)>0 \forall x \in \mathcal{H}\right\} .
$$

We define $X$ by

$$
X=\left\{u \in C\left(\mathbb{R}^{N}, \mathbb{R}\right) \mid u \text { is uniformly continuous and bounded }\right\}
$$

with norm $\|u\|_{X}=\sup _{x \in \mathbb{R}^{N}}|u(x)|$.

Let

and

$$
X^{+}=\left\{u \in X_{i} \mid u(x) \geq 0 \forall x \in \mathcal{H}\right\}
$$




$$
X^{++}=\left\{u \in X_{i}^{+} \mid \inf _{x \in \mathcal{H}} u(x)>0\right\} .
$$

Without occurring confusion, we may write $\|\cdot\|_{X_{p}}$ and $\|\cdot\|_{X}$ as $\|\cdot\|$.

Assume (H1). By general semigroup theory (see Henry, 1981; Pazy, 1983), for any $u_{0} \in X$, (1.2) has a unique local solution $u\left(t, \cdot ; u_{0}\right)$ with $u\left(0, \cdot ; u_{0}\right)=u_{0}(\cdot)$. Moreover, if $u_{0} \in X^{+}$, then $u\left(t, \cdot ; u_{0}\right)$ exist and $u\left(t, \cdot ; u_{0}\right) \in X^{+}$for all $t \geq 0$ (see Proposition 2.2).

Our objective is to explore the spatial spreading dynamics of (1.1) with localized spatial inhomogeneity. The main results of this paper are stated in the following two theorems.

Theorem 1.1 (Positive stationary solutions). Assume (H1) and (H2). Equation (1.2) has a unique stationary solution $u=u^{*}(\cdot) \in X^{++}$. Moreover,

$$
\lim _{r \rightarrow \infty} \sup _{x \in \mathcal{H},\|x\| \geq r}\left|u^{*}(x)-u^{0}\right|=0,
$$

where $u^{0}>0$ is such that $f^{0}\left(u^{0}\right)=0$.

Theorem 1.2 (Stability). Assume (H1) and (H2). For any $u_{0} \in X^{++}$,

$$
\lim _{t \rightarrow \infty}\left\|u\left(t, \cdot ; u_{0}\right)-u^{*}(\cdot)\right\|_{X}=0 .
$$

The rest of the paper is organized as follows. In section 2, we present some preliminary materials to be used in later sections. Section 3 is devoted to the study of positive stationary solutions of (1.1). Theorem 1.1 and Theorem 1.2 are proved in this section.

\section{Preliminary}

In this section, we present some preliminary materials to be used in later sections, including some basic properties of solutions of (1.1); principal eigenvalue theories for spatially periodic dispersal operators with random, and nonlocal; and spatial spreading dynamics of KPP equations in spatially periodic media.

\subsection{Basic Properties of KPP Equations}

In this subsection, we present some basic properties of solutions of (1.1), including comparison principle, global existence, convergence in open compact topology, and decreasing of the so called part metric along the solutions. Throughout this subsection, we assume (H1).

Let $X$ be as in (1.10). For given $u_{0} \in X$, let $u\left(t, \cdot ; u_{0}\right)$ be the (local) solution of (1.2) with $u\left(0, \cdot ; u_{0}\right)=u_{0}(\cdot)$.

Let $X^{+}$and $X^{++}$be as in (1.11) and (1.12). For given $u, v \in X$, we define

$$
u \leq v(u \geq v) \text { if } v-u \in X^{+}\left(u-v \in X^{+}\right)
$$

and

$$
u \ll v(u \gg v) \text { if } v-u \in X^{++}\left(u-v \in X^{++}\right) .
$$

For given continuous and bounded function $u:[0, T) \times \mathbb{R}^{N} \rightarrow \mathbb{R}$, it is called a super-solution (sub-solution) of (1.1) on $[0, T)$ if

$$
u_{t}(t, x) \geq(\leq) \mathcal{A} u(t, x)+u(t, x) f(x, u(t, x)) \quad \forall(t, x) \in(0, T) \times \mathbb{R}^{N} .
$$

Proposition 2.1 (Comparison principle). Assume (H1).

(1) Suppose that $u^{1}(t, x)$ and $u^{2}(t, x)$ are sub-and super-solutions of $(1.1)$ on $[0, T)$ with $u^{1}(0, \cdot) \leq u^{2}(0, \cdot)$. Then $u^{1}(t, \cdot) \leq u^{2}(t, \cdot)$ for $t \in(0, T)$. Moreover, if $u^{1}(0, \cdot) \neq u^{2}(0, \cdot)$, then $u^{1}(t, x)<u^{2}(t, x)$ for $x \in \mathcal{H}$, and $t \in(0, T)$.

(2) If $u_{01}, u_{02} \in X$ and $u_{01} \leq u_{02}, u_{01} \neq u_{02}$, then $u\left(t, x ; u_{01}\right)<u\left(t, x ; u_{02}\right)$ for all $x \in \mathcal{H}$ and $t>0$ at which both $u\left(t, \cdot ; u_{01}\right)$ and $u\left(t, \cdot ; u_{02}\right)$ exist.

(3) If $u_{01}, u_{02} \in X$ and $u_{01} \ll u_{02}$, then $u\left(t, \cdot ; u_{01}\right) \ll u\left(t, \cdot ; u_{02}\right)$ for $t>0$ at which both $u\left(t, \cdot ; u_{01}\right)$ and $u\left(t, \cdot ; u_{02}\right)$ exist.

Proof. (1) The case $v_{2}=0$ follows from comparison principle for parabolic equations. The case $v_{1}=0$ follows from (Shen \& Zhang, 2010) [Propositions 2.1 and 2.2].

(2) follows from (1).

(3) We provide a proof for the case $v_{1}=0$. Other cases can be proved similarly. Take any $T>0$ such that both $u\left(t, \cdot ; u_{01}\right)$ and $u\left(t, \cdot ; u_{02}\right)$ exist on $[0, T]$. It suffices to prove that $u\left(t, \cdot ; u_{02}\right) \gg u\left(t, \cdot ; u_{01}\right)$ for $t \in[0, T]$. To this end, let $w(t, x)=u\left(t, x ; u_{02}\right)-u\left(t, x ; u_{01}\right)$. Then $w(t, x)$ satisfies the following equation, 
where

$$
w_{t}(t, x)=\int_{\mathbb{R}^{N}} \kappa(y-x) w(t, y) d y-w(t, x)+a(t, x) w(t, x),
$$

$$
\begin{aligned}
a(t, x)= & f\left(x, u\left(t, x ; u_{02}\right)\right) \\
& +u\left(t, x ; u_{01}\right) \int_{0}^{1} \partial_{u} f\left(x, s u\left(t, x ; u_{02}\right)+(1-s) u\left(t, x ; u_{01}\right)\right) d s .
\end{aligned}
$$

Let $M>0$ be such that $M \geq \sup _{x \in \mathbb{R}^{N}, t \in[0, T]}(1-a(t, x))$ and $\tilde{w}(t, x)=e^{M t} w(t, x)$. Then $\tilde{w}(t, x)$ satisfies

$$
\tilde{w}_{t}(t, x)=\int_{\mathbb{R}^{N}} \kappa(y-x) \tilde{w}(t, y) d y+[M-1+a(t, x)] \tilde{w}(t, x) .
$$

Let $\mathcal{K}: X \rightarrow X$ be defined by

$$
(\mathcal{K} u)(x)=\int_{\mathbb{R}^{N}} \kappa(y-x) u(y) d y \quad \text { for } \quad u \in X .
$$

Then $\mathcal{K}$ generates an analytic semigroup on $X$ and

$$
\tilde{w}(t, \cdot)=e^{\mathcal{K} t}\left(u_{02}-u_{01}\right)+\int_{0}^{t} e^{\mathcal{K}(t-\tau)}(M-1+a(\tau, \cdot)) \tilde{w}(\tau, \cdot) d \tau .
$$

Observe that $e^{\mathcal{K} t} u_{0} \geq 0$ for any $u_{0} \in X^{+}$and $t \geq 0$ and $e^{\mathcal{K} t} u_{0} \gg 0$ for any $u_{0} \in X^{++}$and $t \geq 0$. Observe also that $u_{02}-u_{01} \in X^{++}$. By (2), $\tilde{w}(\tau, \cdot) \geq 0$ and hence $(M-1+a(\tau, \cdot)) \tilde{w}(\tau, \cdot) \geq 0$ for $\tau \in[0, T]$. It then follows that $\tilde{w}(t, \cdot) \gg 0$ and then $w(t, \cdot) \gg 0$ (i.e. $\left.u\left(t, \cdot ; u_{02}\right) \gg u\left(t, \cdot ; u_{01}\right)\right)$ for $t \in[0, T]$.

Proposition 2.2. Assume $(\mathrm{H} 1)$. For any given $u\left(t, \cdot ; u_{0}\right)$ exists for all $t \geq 0$.

Proof. Let $u_{0} \in X^{+}$be given. There is $M \gg 1$ such that $0 \leq u_{0}(x) \leq M$ and $f(x, M)<0$ for all $x \in \mathcal{H}$. Then by Proposition 2.1,

$$
0 \leq u\left(t, \cdot ; u_{0}\right) \leq M
$$

for any $t>0$ at which $u\left(t, \cdot ; u_{0}\right)$ exists. It is then not difficult to prove that for any $T>0$ such that $u\left(t, \cdot ; u_{0}\right)$ exists on $(0, T), \lim _{t \rightarrow T} u\left(t, \cdot ; u_{0}\right)$ exists in $X$. This implies that $u\left(t, \cdot ; u_{0}\right)$ exists and $u\left(t, \cdot ; u_{0}\right) \geq 0$ for all $t \geq 0$.

For given $u, v \in X^{++}$, define

$$
\rho(u, v)=\inf \left\{\ln \alpha \mid \frac{1}{\alpha} u \leq v \leq \alpha u, \alpha \geq 1\right\} .
$$

Observe that $\rho(u, v)$ is well defined and there is $\alpha \geq 1$ such that $\rho(u, v)=\ln \alpha$. Moreover, $\rho(u, v)=\rho(v, u)$ and $\rho(u, v)=0$ iff $u \equiv v$. In literature, $\rho(u, v)$ is called the part metric between $u$ and $v$.

Proposition 2.3. For given $u_{0}, v_{0} \in X^{++}$with $u_{0} \neq v_{0}, \rho\left(u\left(t, \cdot ; u_{0}\right), u\left(t, \cdot ; v_{0}\right)\right)$ is non-increasing in $t \in(0, \infty)$.

Proof. It can be proved by similar argument in (Kong \& Shen, 2011) [Proposition 3.3]. For completeness, we provide a proof here.

First, note that there is $\alpha^{*}>1$ such that $\rho\left(u_{0}, v_{0}\right)=\ln \alpha^{*}$ and $\frac{1}{\alpha^{*}} u_{0} \leq v_{0} \leq \alpha^{*} u_{0}$. By Proposition 2.1,

$$
u\left(t, \cdot ; v_{0}\right) \leq u\left(t, \cdot ; \alpha^{*} u_{0}\right) \text { for } t>0 \text {. }
$$

Let $v(t, x)=\alpha^{*} u\left(t, x ; u_{0}\right)$. Then

$$
\begin{aligned}
v_{t}(t, x) & =\mathcal{A} v(t, x)+v(t, x) f\left(x, u\left(t, x ; u_{0}\right)\right) \\
& =\mathcal{A} v(t, x)+v(t, x) f(x, v(t, x))+v(t, x) f\left(x, u\left(t, x ; u_{0}\right)\right)-v(t, x) f(x, v(t, x)) \\
& >\mathcal{A} v(t, x)+v(t, x) f(x, v(t, x)) .
\end{aligned}
$$

This together with Proposition 2.1 implies that

$$
u\left(t, \cdot ; \alpha^{*} u_{0}\right) \leq \alpha^{*} u\left(t, \cdot ; u_{0}\right) \text { for } \quad t>0
$$

and then

Similarly, it can be proved that

$$
u\left(t, \cdot ; v_{0}\right) \leq \alpha^{*} u\left(t, \cdot ; u_{0}\right) \text { for } t>0
$$

It then follows that

and hence

$$
\frac{1}{\alpha^{*}} u\left(t, \cdot ; u_{0}\right) \leq u\left(t, \cdot ; v_{0}\right) \text { for } t>0 .
$$

$$
\rho\left(u\left(t, \cdot ; u_{0}\right), u\left(t, \cdot ; v_{0}\right)\right) \leq \rho\left(u_{0}, v_{0}\right) \quad \forall t>0
$$

$$
\rho\left(u\left(t_{2}, \cdot ; u_{0}\right), u\left(t_{2}, \cdot ; v_{0}\right)\right) \leq \rho\left(u\left(t_{1}, \cdot ; u_{0}\right), u\left(t_{1}, \cdot ; v_{0}\right)\right) \quad \forall 0 \leq t_{1}<t_{2}
$$




\subsection{Principal Eigenvalues of Spatially Periodic Dispersal Operators}

In this subsection, we present some principal eigenvalue theories for spatially periodic dispersal operators with hybrid dispersals.

Let $p=\left(p_{1}, p_{2}, \ldots, p_{N}\right)$ with $p_{i}>0$ for $i=1,2, \cdots, N$ and $X_{p}$ be as in (1.7). We will denote $\mathcal{I}$ as an identity map on the Banach space under consideration. For given $\xi \in S^{N-1}, \mu \in \mathbb{R}, a \in X_{p}$, consider the following eigenvalue problems,

$$
\left\{\begin{array}{l}
O u(x)=\lambda u(x), \quad x \in \mathbb{R}^{N} \\
u\left(x+p_{i} \mathbf{e}_{\mathbf{i}}\right)=u(x), \quad x \in \mathbb{R}^{N},
\end{array}\right.
$$

where

$$
O u(x):=v_{1} \Delta u(x)+v_{2} \int_{\mathbb{R}^{N}} e^{-\mu(y-x) \cdot \xi} \kappa(y-x) u(y) d y-2 \mu v_{2} \xi \cdot \nabla u(x)+\left(a(x)+v_{1} \mu^{2}-v_{2}\right) u(x)
$$

and $O: \mathcal{D}(O) \subset X_{p} \rightarrow X_{p}$.

Observe that if $v_{1}=1$, and $v_{2}=0$,

$$
(O u)(x)=\Delta u(x)-2 \mu \xi \cdot \nabla u(x)+\left(a(x)+\mu^{2}\right) u(x) \quad \forall u \in \mathcal{D}(O) \subset X_{p},
$$

If $v_{1}=0$, and $v_{2}=1$,

$$
(O u)(x)=\int_{\mathbb{R}^{N}} e^{-\mu(y-x) \cdot \xi} \kappa(y-x) u(y) d y-u(x)+a(x) u(x) \quad \forall u \in \mathcal{D}(O) \subset X_{p}
$$

Let $\sigma(O)$ be the spectrum of $O$.

Definition 2.1. Let $\mu \in \mathbb{R}$, and $\xi \in S^{N-1}$ be given. A real number $\lambda(\mu, \xi, a) \in \mathbb{R}$ is called the principal eigenvalue of $O$ if it is an isolated algebraic simple eigenvalue of $O$ with a positive eigenfunction and for any $\lambda \in \sigma(O) \backslash\{\lambda(\mu, \xi, a)\}$, $\operatorname{Re} \lambda<\lambda(\mu, \xi, a)$.

For given $\mu \in \mathbb{R}$, and $\xi \in S^{N-1}$, let

$$
\lambda^{0}(\mu, \xi, a)=\sup \{\operatorname{Re} \mu \mid \mu \in \sigma(O)\} .
$$

Observe that for any $\mu \in \mathbb{R}$ and $\xi \in S^{N-1}, O$ generates an analytic semigroup $\{T(t)\}_{t \geq 0}$ in $X_{p}$ and moreover, $T(t)$ is strongly positive (that is, $T(t) u_{0} \geq 0$ for any $t \geq 0$ and $u_{0} \in X_{p}^{+}$and $T(t) u_{0} \gg 0$ for any $t>0$ and $u_{0} \in X_{p}^{+} \backslash\{0\}$ ). Then by (Meyer-Nieberg, 1991) [Proposition 4.1.1], $r(T(t)) \in \sigma(T(t))$ for any $t>0$, where $r(T(t))$ is the spectral radius of $T(t)$. Hence by the spectral mapping theorem (see Chicone \& Latushkin, 1999; [Theorem 2.7]), $\lambda^{0}(\mu, \xi, a) \in \sigma(O)$. Observe also that $\lambda^{0}(0, \xi, a)$ are independent of $\xi \in S^{N-1}$. We may then put

$$
\lambda^{0}(a)=\lambda^{0}(0, \xi, a) .
$$

It is well known that the principal eigenvalue $\lambda(\mu, \xi, a)$ in (2.6) exist for all $\mu \in \mathbb{R}$ and $\xi \in S^{N-1}$ and

$$
\lambda(\mu, \xi, a)=\lambda^{0}(\mu, \xi, a) .
$$

The principal eigenvalue of $O$ in (2.7) may not exist (see Shen \& Zhang, 2010 for examples). If the principal eigenvalue $\lambda(\mu, \xi, a)$ exists in (2.7), then

$$
\lambda(\mu, \xi, a)=\lambda^{0}(\mu, \xi, a) .
$$

Regarding the existence of principal eigenvalue of $O$ in (2.7), the following proposition is proved in (Shen \& Zhang, 2010; Shen \& Zhang, 2012).

Proposition 2.4 (Existence of principal eigenvalue). (1) If $a \in C^{N}\left(\mathbb{R}^{N}, \mathbb{R}\right) \cap X_{p}$ and the partial derivatives of $a(x)$ up to order $N-1$ are zero at some $x_{0}$ satisfying that $a\left(x_{0}\right)=\max _{x \in \mathbb{R}^{N}} a(x)$, then the principal eigenvalue $\lambda(\mu, \xi, a)$ of $O$ exists for all $\mu \in \mathbb{R}$ and $\xi \in S^{N-1}$.

(2) If a (x) satisfies that $\max _{x \in \mathbb{R}^{N}} a(x)-\min _{x \in \mathbb{R}^{N}} a(x)<\inf _{\xi \in S^{N-1}} \int_{z \xi \xi 0} k(z) d z$, then the principal eigenvalue $\lambda(\mu, \xi, a)$ of $O$ exists for all $\mu \in \mathbb{R}$ and $\xi \in S^{N-1}$.

Proof. (1) It follows from (Shen \& Zhang, 2010) [Theorem B].

(2) It follows from (Shen \& Zhang, 2012) [Theorem B']. 
Let $\hat{a}$ be the average of $a(\cdot)$, that is,

$$
\hat{a}=\frac{1}{|D|} \int_{D} a(x) d x \text { for }
$$

where

$$
D=\left[0, p_{1}\right] \times\left[0, p_{2}\right] \times \cdots \times\left[0, p_{N}\right] \cap \mathcal{H}
$$

and

$$
|D|=p_{1} \times p_{2} \times \cdots \times p_{N} \text { for }
$$

By Proposition $2.4(2), \lambda(\mu, \xi, \hat{a})$ exists for all $\mu \in \mathbb{R}$ and $\xi \in S^{N-1}$. The following proposition shows a relation between $\lambda^{0}(\mu, \xi)$ and $\lambda^{0}(\mu, \xi, \hat{a})$.

Proposition 2.5 (Influence of spatial variation). For given $\mu \in \mathbb{R}$, and $\xi \in S^{N-1}$, there holds

$$
\lambda^{0}(\mu, \xi) \geq \lambda^{0}(\mu, \xi, \hat{a}) .
$$

Proof. It follow from (Hetzer, Shen, \& Zhang, 2013) [Theorem 2.1].

We remark that $\lambda(\mu, \xi, \hat{a})\left(=\lambda^{0}(\mu, \xi, \hat{a})\right)$ have the following explicit expressions,

$$
\lambda(\mu, \xi, \hat{a})=v_{1} \mu^{2}+v_{2}\left(\int_{\mathbb{R}^{N}} e^{-\mu z \cdot \xi} \kappa(z) d z-1\right)+\hat{a}
$$

\subsection{KPP Equations in Spatially Periodic Media}

In this subsection, we recall some spatial spreading dynamics of KPP equations in spatially periodic media.

Consider

$$
u_{t}(t, x)=\mathcal{A} u(t, x)+u(t, x) g(x, u(t, x)), \quad x \in \mathbb{R}^{N},
$$

where $g(\cdot, \cdot)$ are periodic in the first variable and monostable in the second variable. More precisely, we assume

(P1) $g: \mathcal{H} \times \mathbb{R} \rightarrow \mathbb{R}$ is a $C^{2}$ function, $g\left(x+p_{l} \mathbf{e}_{l}, u\right)=g(x, u)$, where $p_{l}>0$ and $g(x, u)<0$ for all $(x, u) \in \mathcal{H} \times \mathbb{R}^{+}$with $u \geq \alpha_{0}$ for some $\alpha_{0}>0$ and $\partial_{u} g(x, u)<0$ for all $(x, u) \in \mathcal{H} \times \mathbb{R}^{+}$.

(P2) $\lambda^{0}(g(\cdot, 0))>0$.

Assume (P1). Similarly, by general semigroup theory, for any $u_{0} \in X,(2.13)$ has a unique (local) solution $u\left(t, \cdot ; u_{0}, g(\cdot, \cdot)\right)(\in$ $X$ ) with initial data $u_{0}(\cdot)$. Moreover, if $u_{0} \in X_{p}$, then $u\left(t, \cdot ; u_{0}, g(\cdot, \cdot)\right) \in X_{p}$ for any $t>0$ at which $u\left(t, \cdot ; u_{0}, g(\cdot, \cdot)\right)$ exists. By Proposition 2.1, if $u_{0} \in X^{+}$, then $u\left(t, \cdot ; u_{0}, g(\cdot, \cdot)\right)$ exists and $u\left(t, \cdot ; u_{0}, g(\cdot, \cdot)\right) \in X^{+}$for all $t>0$.

Proposition 2.6 (Spatially periodic positive stationary solution). Assume (P1) and (P2). Then (2.13) has a unique spatially periodic stationary solution $u^{*}(\cdot ; g(\cdot, \cdot)) \in X_{p}^{++}$which is globally asymptotically stable with respect to perturbations in $X_{p}^{+} \backslash\{0\}$.

Proof. It follows from (Zhao, 1996) [Theorem 2.3] and (Shen, \& Zhang, 2012) [Theorem C].

Let $\hat{g}(u)$ be the spatial average of $g(x, u)$, that is,

$$
\hat{g}(u)=\frac{1}{|D|} \int_{D} g(x, u) d x \text { for }
$$

where $D,|D|$ is as in (2.10).

Assume

(P3) $\hat{g}(0)>0$.

Observe that $\lambda(\hat{g}(0))=\hat{g}(0)$. Then by Proposition 2.5, (P3) implies (P2). 


\section{Positive Stationary Solutions}

In this section, we explore the existence of positive stationary solutions of (1.1), and prove Theorem 1.1 and 1.2.

Throughout this section, we assume (H1) and (H2). We first prove some lemmas.

Lemma 3.1. For any $\epsilon>0$, there are $p=\left(p_{1}, p_{2}, \cdots, p_{N}\right) \in \mathbb{N}^{N}$ and $h \in X_{p} \cap C^{N}(\mathcal{H}, \mathbb{R})$ such that

$$
\begin{gathered}
f(x, 0) \geq h(x) \quad \text { for } \quad x \in \mathcal{H}, \\
\left.\hat{h} \geq f^{0}(0)-\epsilon \quad \text { (hence } \quad \lambda^{0}(h(\cdot)) \geq f^{0}(0)-\epsilon\right),
\end{gathered}
$$

and the partial derivatives of $h(x)$ up to order $N-1$ are zero at some $x_{0} \in \mathcal{H}$ with $h\left(x_{0}\right)=\max _{x \in \mathcal{H}} h(x)$, where $\hat{h}$ is the average of $h(\cdot)$ (see (2.9) for the definition).

Proof. By (H2), there is $L_{0}>0$ such that $f(x, 0)=f^{0}(0)$ for $x \in \mathcal{H}$ with $\|x\| \geq L_{0}$. Let $M_{0}=\inf _{x \in \mathcal{H}} f(x, 0)$. Let $h_{0}: \mathbb{R} \rightarrow$ $[0,1]$ be a smooth function such that $h_{0}(s)=1$ for $|s| \leq 1$ and $h_{0}(s)=0$ for $|s| \geq 2$. For any $p=\left(p_{1}, p_{2}, \cdots, p_{N}\right) \in \mathbb{N}^{N}$ with $p_{j}>4 L_{0}$, let $h \in X_{p} \cap C^{N}(\mathcal{H}, \mathbb{R})$ be such that

$$
h(x)=f^{0}(0)-h_{0}\left(\frac{\|x\|^{2}}{L_{0}^{2}}\right)\left(f^{0}(0)-M_{0}\right) \quad \text { for } \quad x \in\left(\left[-\frac{p_{1}}{2}, \frac{p_{1}}{2}\right] \times\left[-\frac{p_{2}}{2}, \frac{p_{2}}{2}\right] \times \cdots \times\left[-\frac{p_{N}}{2}, \frac{p_{N}}{2}\right]\right) \cap \mathcal{H} .
$$

Then

$$
f(x, 0) \geq h(x) \quad \forall x \in \mathcal{H} .
$$

It is clear that the partial derivatives of $h(x)$ up to order $N-1$ are zero at some $x_{0} \in \mathcal{H}$ with $h\left(x_{0}\right)=\max _{x \in \mathcal{H}} h(x)\left(=f^{0}(0)\right)$. For given $\epsilon>0$, choosing $p_{j} \gg 1$, we have

$$
\hat{h}>f^{0}(0)-\epsilon .
$$

By Proposition 2.5, $\lambda^{0}(h(\cdot)) \geq \lambda^{0}(\hat{h})=\hat{h}$ and hence

$$
\lambda^{0}(h(\cdot)) \geq f^{0}(0)-\epsilon
$$

The lemma is thus proved.

Lemma 3.2. Suppose that $\tilde{u}^{*}: \mathbb{R}^{N} \rightarrow\left[\sigma_{0}, M_{0}\right]$ is Lebesgue measurable, where $\sigma_{0}$ and $M_{0}$ are two positive constants. If

$$
\Delta \tilde{u}^{*}(x)+\int_{\mathbb{R}^{N}} \kappa(y-x) \tilde{u}^{*}(y) d y-\tilde{u}^{*}(x)+\tilde{u}^{*}(x) \tilde{f}\left(x, \tilde{u}^{*}(x)\right)=0 \quad \forall x \in \mathbb{R}^{N},
$$

where $\tilde{f}(x, u)=f(x, u)$ or $f^{0}(u)$ for all $x \in \mathbb{R}^{N}$ and $u \in \mathbb{R}$, then $\tilde{u}^{*}(\cdot) \in X^{++}$.

Proof. We prove the case that $\tilde{f}(x, u)=f(x, u)$. The case that $\tilde{f}(x, u)=f^{0}(u)$ can be proved similarly.

Let $h^{*}(x)=\Delta \tilde{u}^{*}(x)+\int_{\mathbb{R}^{N}} \kappa(y-x) \tilde{u}^{*}(y) d y$ for $x \in \mathbb{R}^{N}$. Then $h^{*}(\cdot)$ is $C^{1}$ and has bounded first order partial derivatives. Let

$$
F(x, \alpha)=h^{*}(x)-\alpha+\alpha f(x, \alpha) \quad \forall x \in \mathbb{R}^{N}, \alpha \in \mathbb{R} .
$$

Then $F: \mathbb{R}^{N} \times \mathbb{R} \rightarrow \mathbb{R}$ is $C^{1}$ and $F\left(x, \tilde{u}^{*}(x)\right)=0$ for each $x \in \mathbb{R}^{N}$. If $\alpha^{*}>0$ is such that $F\left(x, \alpha^{*}\right)=0$, then

$$
-1+f\left(x, \alpha^{*}\right)=-\frac{h^{*}(x)}{\alpha^{*}}<0
$$

and hence

$$
\partial_{\alpha} F\left(x, \alpha^{*}\right)=-1+f\left(x, \alpha^{*}\right)+\alpha^{*} \partial_{u} f\left(x, \alpha^{*}\right)<0 .
$$

By Implicit Function Theorem, $\tilde{u}^{*}(x)$ is $C^{1}$ in $x$. Moreover,

$$
\frac{\partial \tilde{u}^{*}(x)}{\partial x_{j}}=\frac{\frac{\partial h^{*}(x)}{\partial x_{j}}}{-1+f\left(x, \tilde{u}^{*}(x)\right)+\partial_{u} f\left(x, \tilde{u}^{*}(x)\right) \tilde{u}^{*}(x)} \quad \forall x \in \mathbb{R}^{N}, 1 \leq j \leq N .
$$

Therefore, $\tilde{u}^{*}$ has bounded first order partial derivatives. It then follows that $\tilde{u}^{*}(x)$ is uniformly continuous in $x \in \mathbb{R}^{N}$ and then $\tilde{u}^{*} \in X^{++}$.

Lemma 3.3. Suppose that $u^{*}(\cdot) \in X^{++}$and $u=u^{*}(\cdot)$ is a stationary solution of $(1)$. Then

$$
u^{*}(x) \rightarrow u^{0} \quad \text { as } \quad\|x\| \rightarrow \infty .
$$


Proof. Assume that $u^{*}(x) \nrightarrow u^{0}$ as $\|x\| \rightarrow \infty$. Then there are $\epsilon_{0}>0$ and $x_{n} \in \mathbb{R}^{N}$ such that $\left\|x_{n}\right\| \rightarrow \infty$ and

$$
\left|u^{*}\left(x_{n}\right)-u^{0}\right| \geq \epsilon_{0} \text { for } n=1,2, \cdots .
$$

By the uniform continuity of $u^{*}(x)$ in $x \in \mathbb{R}^{N}$, without loss of generality, we may assume that there is a continuous function $\tilde{u}^{*}: \mathbb{R}^{N} \rightarrow\left[\sigma_{0}, M_{0}\right]$ for some $\sigma_{0}, M_{0}>0$ such that

$$
u\left(x+x_{n}\right) \rightarrow \tilde{u}^{*}(x)
$$

as $n \rightarrow \infty$ uniformly in $x$ on bounded sets. By the Lebesgue Dominated Convergence Theorem, we have

$$
\Delta \tilde{u}^{*}(x)+\int_{\mathbb{R}^{N}} \kappa(y-x) \tilde{u}^{*}(y) d y-\tilde{u}^{*}(x)+\tilde{u}^{*}(x) f^{0}\left(\tilde{u}^{*}(x)\right)=0 \quad \forall x \in \mathbb{R}^{N} .
$$

By Lemma 3.2, $\tilde{u}^{*} \in X^{++}$. By Proposition 2.6 again, we have $\tilde{u}^{*}(x) \equiv u^{0}$ and then $u^{*}\left(x_{n}\right) \rightarrow u^{0}$ as $n \rightarrow \infty$. This is a contradiction. Therefore $u^{*}(x) \rightarrow u^{0}$ as $\|x\| \rightarrow \infty$.

Lemma 3.4. There is $u^{-} \in X^{++}$such that for any $\delta>0$ sufficiently small, $u\left(t, x ; \delta u^{-}\right)$is increasing in $t>0$ and $u^{-, *, \delta} \in X^{++}$, where $u^{-, *, \delta}(x)=\lim _{t \rightarrow \infty} u\left(t, x ; \delta u^{-}\right)$, and hence $u=u^{-, *, \delta}(\cdot)$ is a stationary solution of $(1.1)$ in $X^{++}$.

Proof. Let $M^{*}>0$ be such that $f\left(x, M^{*}\right)<0$. Let $\epsilon>0$ be such that

$$
f^{0}(0)-\epsilon>0
$$

By Lemma 3.1, there are $p \in \mathbb{N}^{N}$ and $h(\cdot) \in X_{p} \cap C^{N}(\mathcal{H}, \mathbb{R})$ such that

$$
f(x, 0) \geq h(x), \text { and } \hat{h} \geq f^{0}(0)-\epsilon(>0) .
$$

Moreover, the partial derivatives of $h(x)$ up to order $N-1$ are zero at some $x_{0} \in \mathcal{H}$ with $h\left(x_{0}\right)=\max _{x \in \mathcal{H}} h(x)$. Let $u^{-}$ be the positive principal eigenfunction of $O$ with $a(\cdot)=h(\cdot)$ and $\left\|u^{-}\right\|=1$. It is not difficult to verify that $u=\delta u^{-}$is a sub-solution of (1.1) for any $\delta>0$ sufficiently small. It then follows that for any $\delta>0$ sufficiently small,

$$
\delta u^{-}(\cdot) \leq u\left(t_{1}, \cdot ; \delta u^{-}\right) \leq u\left(t, \cdot ; \delta u^{-}\right) \quad \forall 0<t_{1}<t .
$$

This implies that there is a Lebesgue measurable function $u^{-, *, \delta}: \mathcal{H} \rightarrow\left[\sigma_{0}, M_{0}\right]$ for some $\sigma_{0}, M_{0}>0$ such that

$$
\lim _{t \rightarrow \infty} u\left(t, x ; \delta u^{-}\right)=u^{-, *, \delta}(x) \quad \forall x \in \mathcal{H} .
$$

By Lemma 3.2, $u^{-, *, \delta} \in X^{++}$. Therefore $u^{-, *, \delta} \in X^{++}$and $u=u^{-, *, \delta}(\cdot)$ is a stationary solution of (1.1) in $X^{++}$.

Lemma 3.5. Let $M \gg 1$ be such that $f(x, M)<0$ for $x \in \mathcal{H}$. Then $\lim _{t \rightarrow \infty} u\left(t, x ; u_{0}\right)$ exists for every $x \in \mathcal{H}$, where $u_{0}(x) \equiv M$. Moreover, $u^{+, *, M}(\cdot) \in X^{++}$, where $u^{+, *, M}(x):=\lim _{t \rightarrow \infty} u\left(t, x ; u_{0}\right)$, and hence $u=u^{+, *, M}(\cdot)$ is a stationary solution of (1.1) in $X^{++}$.

Proof. For any $M>1$ with $f(x, M)<0$ for all $x \in \mathcal{H}, u=M$ is a super-solution of (1). Hence

$$
u(t, \cdot ; M) \leq u\left(t_{1}, \cdot ; M\right) \leq M \quad \forall 0 \leq t_{1}<t .
$$

It then follows that $\lim _{t \rightarrow \infty} u(t, x ; M)$ exists for all $x \in \mathbb{R}^{N}$. Let $u^{+, *, M}(x)=\lim _{t \rightarrow \infty} u(t, x ; M)$. We have $u^{+, *, M}(x) \geq u^{-, *, \delta}(x)$ for $0<\delta \ll 1$. By the similar arguments as in Lemma 3.4, $u^{+, *, M} \in X^{++}$and $u=u^{+, *, M}(\cdot)$ is a stationary solution of (1.1) in $X^{++}$.

Proof of Theorem 1.1. (1) First, by Lemmas 3.4 and 3.5, (1) has stationary solutions in $X^{++}$. We claim that stationary solution of (1) in $X^{++}$is unique. In fact, suppose that $u^{1, *}$ and $u^{2, *}$ are two stationary solutions of (1) in $X^{++}$. Assume that $u^{1, *} \neq u^{2, *}$. Then there is $\alpha^{*}>1$ such that $\rho\left(u^{1, *}, u^{2, *}\right)=\ln \alpha^{*}>0$. Note that

$$
\frac{1}{\alpha^{*}} u^{1, *} \leq u^{2, *} \leq \alpha^{*} u^{1, *} .
$$

By Lemma 3.3, $\lim _{\|x\| \rightarrow \infty} u^{1, *}(x)=u^{0}$ and $\lim _{\|x\| \rightarrow \infty} u^{2, *}(x)=u^{0}$. This implies that there is $\epsilon>0$ such that

$$
\frac{1}{\alpha^{*}-\epsilon} u^{1, *}(x) \leq u^{2, *}(x) \leq\left(\alpha^{*}-\epsilon\right) u^{1, *}(x) \quad \text { for } \quad\|x\| \gg 1 .
$$

By Proposition 2.1 and the arguments in Proposition 2.3,

It then follows that for $0<\epsilon \ll 1$,

$$
\frac{1}{\alpha^{*}} u^{1, *}(x)<u^{2, *}(x)<\alpha^{*} u^{1, *}(x) \quad \forall x \in \mathbb{R}^{N} .
$$

$$
\frac{1}{\alpha^{*}-\epsilon} u^{1, *}(x) \leq u^{2, *}(x) \leq\left(\alpha^{*}-\epsilon\right) u^{1, *}(x) \quad \forall x \in \mathbb{R}^{N}
$$

and then $\rho\left(u^{1, *}, u^{2, *}\right) \leq \ln \left(\alpha^{*}-\epsilon\right)$, this is a contradiction. Therefore $u^{1, *}=u^{2, *}$ and (1.1) has a unique stationary solution $u^{*}$ in $X^{++}$. 
Proof of Theorem 1.2. For any $u_{0} \in X^{++}$, there is $\delta>0$ sufficiently small and $M>0$ sufficiently large such that $\delta u^{-} \leq$ $u_{0} \leq M$ and $u=\delta u^{-}$is a sub-solution of (1) ( $u^{-}$is as in Lemma 3.4) and $u=M$ is a super-solution of (1.1). Then

$$
\delta u^{-} \leq u\left(t, \cdot ; \delta u^{-}\right) \leq u\left(t, \cdot ; u_{0}\right) \leq u(t, \cdot ; M) \leq M \quad \forall t \geq 0 .
$$

By Theorem 1.1, Lemmas 3.4 and 3.5, and Dini's Theorem,

$$
u\left(t, x ; \delta u^{-}\right)<u^{*}(x)<u(t, x ; M) \quad \forall t>0, x \in \mathcal{H}
$$

and

$$
\lim _{t \rightarrow \infty} u\left(t, x ; \delta u^{-}\right)=\lim _{t \rightarrow \infty} u(t, x ; M)=u^{*}(x)
$$

uniformly in $x$ on bounded sets. It then follows that

$$
\lim _{t \rightarrow \infty} u\left(t, x ; u_{0}\right)=u^{*}(x)
$$

uniformly in $x$ on bounded sets.

We claim that $\left\|u\left(t, \cdot ; u_{0}\right)-u^{*}(\cdot)\right\| \rightarrow 0$ as $t \rightarrow \infty$. Assume the claim is not true. Then there are $\epsilon_{0}>0, t_{n} \rightarrow \infty$, and $x_{n}$ with $\left\|x_{n}\right\| \rightarrow \infty$ such that

Then by Lemma 3.3,

$$
\left|u\left(t_{n}, x_{n} ; u_{0}\right)-u^{*}\left(x_{n}\right)\right| \geq \epsilon_{0} \quad \forall n \in \mathbb{N} .
$$

$$
\left|u\left(t_{n}, x_{n} ; u_{0}\right)-u^{0}\right| \geq \frac{\epsilon_{0}}{2} \quad \forall n \gg 1 .
$$

Let $\tilde{\delta}>0$ and $\tilde{M}>0$ be such that

$$
\tilde{\delta} \leq u\left(t, \cdot ; u_{0}\right) \leq \tilde{M} \quad \forall t \geq 0 .
$$

For any $\epsilon>0$, let $T>0$ be such that

$$
\left|u\left(T, \cdot ; \tilde{\delta}, f^{0}(\cdot)\right)-u^{0}\right|<\epsilon, \quad\left|u\left(T, \cdot ; \tilde{M}, f^{0}(\cdot)\right)-u^{0}\right|<\epsilon .
$$

Observe that

and

$$
\tilde{\delta} \leq u\left(t_{n}-T, x_{n}+x ; u_{0}\right) \leq \tilde{M}
$$

$u\left(t_{n}, x_{n}+\cdot ; u_{0}\right)=u\left(T, x_{n}+\cdot ; u\left(t_{n}-T, \cdot ; u_{0}\right)\right)=u\left(T, \cdot ; u\left(t_{n}-T, \cdot+x_{n} ; u_{0}\right), f\left(\cdot+x_{n}, \cdot\right)\right)$

for $n \gg 1$. Then

$$
u\left(T, \cdot ; \tilde{\delta}, f\left(\cdot+x_{n}\right)\right) \leq u\left(t_{n}, x_{n}+\cdot ; u_{0}\right) \leq u\left(T, \cdot ; \tilde{M}, f\left(\cdot+x_{n}, \cdot\right)\right) .
$$

Observe also that $f\left(x+x_{n}, u\right) \rightarrow f^{0}(u)$ as $n \rightarrow \infty$ uniformly in $(x, u)$ on bounded sets. Then

$$
u\left(T, x ; \tilde{\delta}, f\left(\cdot+x_{n}, \cdot\right)\right) \rightarrow u\left(T, x ; \tilde{\delta}, f^{0}(\cdot)\right)
$$

and

$$
u\left(T, x ; \tilde{M}, f\left(\cdot+x_{n}, \cdot\right)\right) \rightarrow u\left(T, x ; \tilde{M}, f^{0}(\cdot)\right)
$$

as $n \rightarrow \infty$ uniformly in $x$ on bounded sets. This together with (3.1) implies that

$$
\left|u\left(T, 0 ; \tilde{\delta}, f\left(\cdot+x_{n}, \cdot\right)\right)-u^{0}\right|<2 \epsilon, \quad\left|u\left(T, 0 ; \tilde{M}, f\left(\cdot+x_{n}, \cdot\right)\right)-u^{0}\right|<2 \epsilon \quad \text { for } \quad n \gg 1
$$

and then by (3.2),

$$
\left|u\left(t_{n}, x_{n} ; u_{0}\right)-u^{0}\right|<2 \epsilon \text { for } n \gg 1 .
$$

Hence $\lim _{n \rightarrow \infty} u\left(t_{n}, x_{n} ; u_{0}\right)=u^{0}$, which is a contradiction. Therefore $\left\|u\left(t, \cdot ; u_{0}\right)-u^{*}(\cdot)\right\| \rightarrow 0$ as $t \rightarrow \infty$.

\section{Acknowledgements}

The author thanks the referees for valuable comments and suggestions which improved the presentation considerably.

\section{References}

Aronson, D. G., \& Weinberger, H. F. (1957). Nonlinear diffusion in population genetics, combustion, and nerve pulse propagation, in "Partail Differential Equations and Related Topics" (J. Goldstein, Ed.), Lecture Notes in Math., 466, Springer-Verlag, New York, 5-49. http://dx.doi.org/10.1007/BFb0070595

Aronson, D. G. \& Weinberger, H. F. (1978). Multidimensional nonlinear diffusions arising in population genetics, $A d v$. Math., 30, 33-76. http://dx.doi.org/10.1016/0001-8708(78)90130-5

Bates, P. \& Zhao, G. (2007). Existence, uniqueness and stability of the stationary solution to a nonlocal evolution equation arising in population dispersal, J. Math. Anal. Appl., 332, 428-440. http://dx.doi.org/10.1016/j.jmaa.2006.09.007

Berestycki, H., Hamel, F., \& Nadirashvili, N. (2005). The speed of propagation for KPP type problems, I - Periodic framework, J. Eur. Math. Soc., 7, 172-213. http://dx.doi.org/10.1007/s00285-004-0313-3 
Berestycki, H., Hamel, F., \& Nadirashvili, N. (2010). The speed of propagation for KPP type problems, II - General domains, J. Amer. Math. Soc., 23, 1-34. http://dx.doi.org/10.1090/S0894-0347-09-00633-X

Berestycki, H., Hamel, F., \& Roques, L. (2005). Analysis of periodically fragmented environment model: II - Biological invasions and pulsating traveling fronts, J. Math. Pures Appl., 84, 1101-1146.

Berestycki, H., \& Nadin, G. (2016). Asymptotic spreading for general heterogeneous Fisher-KPP type equations, preprint. http://lodel.ehess.fr/cams/docannexe.php?id=1344

Berestycki, H., Jin, T., \& Silvestre, L. (2016). Propagation in a nonlocal reaction diffusion equation with spatial and genetic trait structure, Nonlinearity, 29(4), 1434-1466. http://dx.doi.org/10.1088/0951-7715/29/4/1434

Cantrell, R. S., \& Cosner, C. (2003). Spatial Ecology via Reactiond-Diffusion Equations, Series in Mathematical and Computational Biology, John Wiley and Sons, Chichester, UK. http://dx.doi.org/10.1002/0470871296.ch1

Chasseigne, E., Chaves, M., \& Rossi, J. D. (2006). Asymptotic behavior for nonlocal diffusion equations, J. Math. Pures Appl. 86, 271-291. http://dx.doi.org/10.1016/j.matpur.2006.04.005

Cortazar, C., Coville, J., Elgueta, M. \& Martinez, S. (2007). A nonlocal inhomogeneous dispersal process, J. Differential Equations, 241, 332-358. http://dx.doi.org/10.1016/j.jde.2007.06.002

Cousens, R., Dytham, C. \& Law, R (2008). Dispersal in plants: a population perspective, Oxford University Press, Oxford. http://dx.doi.org/10.1093/aob/mcn243

Chicone, C. \& Latushkin, Y. (1999). Evolution Semigroups in Dynamical Systems and Differential Equations, Mathematical Surveys and Monographs, 70, American Mathematical Society. http://dx.doi.org/10.1090/surv/070

Coville, J. \& Dupaigne, L. (2005). Propagation speed of travelling fronts in non local reaction-diffusion equations, Nonlinear Analysis, 60, 797-819. http://dx.doi.org/10.1016/j.na.2003.10.030

Coville, J., Dávila, J., \& Martínez, S. (2008). Existence and uniqueness of solutions to a nonlocal equation with monostable nonlinearity, SIAM J. Math. Anal., 39, 1693-1709. http://dx.doi.org/10.1137/060676854

Fife, P. C. (1979). Mathematical Aspects of Reacting and Diffusing Systems, Lecture Notes in Biomathematics, 28, Springer-Verlag, Berlin-New York. http://dx.doi.org/10.1007/978-3-642-93111-6

Fife, P. C. (2003). Some nonclassical trends in parabolic and parabolic-like evolutions. Trends in nonlinear analysis, 153-191, Springer, Berlin.

Fife, P. C., \& Peletier, L. A. (1977). Nonlinear diffusion in population genetics. Arch. Rational Mech Anal., 64, 93-109.

Fisher, R. (1937). The wave of advance of advantageous genes. Ann. of Eugenics, 7, 335-369. http://dx.doi.org/10.1111/j.1469-1809.1937.tb02153.x

Freidlin, M., \& Gärtner, J. (1979). On the propagation of concentration waves in periodic and random media. Soviet Math. Dokl., 20, 1282-1286.

Grinfeld, M., Hines, G., Hutson, V., Mischaikow, K., \& Vickers, G. T. (2005). Non-local dispersal. Differential Integral Equations, 18, 1299-1320.

Hamel, F. (2008). Qualitative properties of monostable pulsating fronts: exponential decay and monotonicity, J. Math. Pures Appl., 89, 355-399. http://dx.doi.org/10.1016/j.matpur.2007.12.005

Henry, D. (1981). Geometric Theory of Semilinear Parabolic Equations. Lecture Notes in Math., 840, Springer-Verlag, Berlin. http://dx.doi.org/10.1007/BFb0089647

Hetzer, G., Shen, W., \& Zhang, A. (2013). Effects of spatial variations and dispersal strategies on principal eigenvalues of dispersal operators and spreading speeds of monostable equations. Rocky Mountain Journal of Mathematics, 43, 489-513. http://dx.doi.org/10.1216/RMJ-2013-43-2-489

Hudson, W., \& Zinner, B. (1995). Existence of traveling waves for reaction diffusion equations of Fisher type in periodic media, Boundary value problems for functional-differential equations, 187-199, World Sci. Publ., River Edge, NJ, 1995. http://dx.doi.org/10.1006/jmaa.1996.0137

Hutson, V., Martinez, S. , Mischaikow, K., \& Vickers, G.T. (2003). The evolution of dispersal. J. Math. Biol., 47,483-517. http://dx.doi.org/10.1007/s00285-003-0210-1

Kametaka, Y. (1976). On the nonlinear diffusion equation of Kolmogorov-Petrovskii- Piskunov type. Osaka J. Math., 13, 
11-66.

Kao, C.-Y., Lou, Y., \& Shen, W. (2010). Evolution of Mixed Dispersal in Periodic Environments. Discrete and Continuous Dynamical Systems, 26, 551-596. http://dx.doi.org/10.3934/dcdsb.2012.17.2047

Kao, C.-Y., Lou, Y., \& Shen, W. (2012). Random dispersal vs non-Local dispersal. Discrete and Continuous Dynamical Systems, Series B, 17, 2047-2072. http://dx.doi.org/10.3934/dcds.2010.26.551

Kolmogorov, A., Petrowsky, I. , \& Piscunov, N. (1937). A study of the equation of diffusion with increase in the quantity of matter, and its application to a biological problem. Bjul. Moskovskogo Gos. Univ., 1 1-26.

Kong, L., \& Shen, W. (2011). Positive Stationary Solutions and Spreading Speeds of KPP Equations in Locally Spatially Inhomogeneous Media. Methods and Applications of Analysis, 18, 427-456.

http://dx.doi.org/10.4310/MAA.2011.v18.n4.a5

Kong, L., \& Shen, W. (2014). Liouville Type Property and Spreading Speeds of KPP Equations in Periodic Media with Localized Spatial Inhomogeneity. Journal of Dynamics and Differential Equations, 26, Issue 1, pp 181-215. http://dx.doi.org/10.1007/s10884-014-9351-8

Lee, C. T., Hoopes, M. F., Diehl, J., Gilliland, W., Huxel, G., Leaver, E. V., McCain, K., Umbanhowar, J., \& Mogilner, A. (2001). Non-local concepts and models in biology. J. theor. Biol., 210 , 201-219. http://dx.doi.org/10.1006/jtbi.2000.2287

Levin, S.A., Muller-Landau, H.C., Nathan, R., \& Chave, J. (2003). The ecology and evolution of seed dispersal: a theoretical perspective. Annu. Rev. Eco. Evol. Syst., 34, 575-604. http://dx.doi.org/10.1146/annurev.ecolsys.34.011802.132428

Li, W.-T., Sun, Y.-J., \& Wang, Z.-C. (2010). Entire solutions in the Fisher-KPP equation with nonlocal dispersal. Nonlinear Anal. Real World Appl., 11, 2302-2313. http://dx.doi.org/10.1016/j.nonrwa.2009.07.005

Liang, X., \& Zhao, X.-Q. (2007). Asymptotic speeds of spread and traveling waves for monotone semiflows with applications. Comm. Pure Appl. Math., 60, 1-40. http://dx.doi.org/10.1002/cpa.20154

Liang, X., Yi, Y., \& Zhao, X.-Q. (2006). Spreading speeds and traveling waves for periodic evolution systems. J. Diff. Eq., 231, 57-77. http://dx.doi.org/10.1016/j.jde.2006.04.010

Murray, J. D. (1989). Mathematical Biology, Springer-Verlag, New York. http://dx.doi.org/10.1007/b98868

Nadin, G. (2009). Traveling fronts in space-time periodic media. J. Math. Pures Appl., 9 (92), $232-262$. http://dx.doi.org/10.1016/j.matpur.2009.04.002

Meyer-Nieberg, P. (1991). Banach Lattices, Springer-Verlag. http://dx.doi.org/10.1007/978-3-642-76724-1

Nolen, J., Rudd, M., \& Xin, J. (2005). Existence of KPP fronts in spatially-temporally periodic adevction and variational principle for propagation speeds. Dynamics of PDE, 2, 1-24. http://dx.doi.org/10.4310/DPDE.2005.v2.n1.a1

Nolen, J., \& Xin, J. (2005). Existence of KPP type fronts in space-time periodic shear flows and a study of minimal speeds based on variational principle. Discrete and Continuous Dynamical Systems, 13, 1217-1234. http://dx.doi.org/10.3934/dcds.2005.13.1217

Nolen, J., Roquejoffre, J-M, \& Ryzhik, L. (2015). Power-Like Delay in Time Inhomogeneous Fisher-KPP Equations. Communications in Partial Differential Equations, 40 (3), 475-505. http://dx.doi.org/10.1080/03605302.2014.972744

Pazy, A. (1983). Semigroups of Linear Operators and Applications to Partial Differential Equations, Springer-Verlag New York Berlin Heidelberg Tokyo. http://dx.doi.org/10.1007/978-1-4612-5561-1

Sattinger, D. H. (1976). On the stability of waves of nonlinear parabolic systems. Advances in Math., 22, $312-355$. http://dx.doi.org/10.1016/0001-8708(76)90098-0

Shen, W. (2011). Existence, uniqueness, and stability of generalized traveling waves in time dependent monostable equations. Journal of Dynamics and Differential Equations, 23, 1-44. http://dx.doi.org/10.1007/s10884-010-9200-3

Shen, W., \& Zhang, A. (2010). Spreading speeds for monostable equations with nonlocal dispersal in space periodic habitats. Journal of Differential Equations, 249, 749-795. http://dx.doi.org/10.1016/j.jde.2010.04.012

Shen, W., \& Zhang, A. (2012). Stationary solutions and spreading speeds of nonlocal monostable equations in space periodic habitats. Proceedings of the American Mathematical Society 140, 1681-1696. http://dx.doi.org/10.1090/S00029939-2011-11011-6 
Shigesada, N., \& Kawasaki, K. (1997). Biological Invasions: Theory and Practice, Oxford University Press.

Skellam, J. G. (1951). Random dispersal in theoretical populations. Biometrika, 38, 196-218.

Uchiyama, K. (1978). The behavior of solutions of some nonlinear diffusion equations for large time. J. Math. Kyoto Univ., 183, 453-508.

Weinberger, H. F. (1982). Long-time behavior of a class of biology models. SIAM J. Math. Anal., 13, 353-396. http://dx.doi.org/10.1137/0513028

Weinberger, H. F. (2002). On spreading speeds and traveling waves for growth and migration models in a periodic habitat. J. Math. Biol., 45, 511-548. http://dx.doi.org/10.1007/s00285-002-0169-3

Zhang, A. (2013). Traveling wave solutions with mixed dispersal for spatially periodic Fisher-KPP equations. Discrete Contin. Dyn. Syst. Suppl., 815-824.

Zhao, X. Q. (1996). Global attractivity and stability in some monotone discrete dynamical systems, Bull. Austral. Math. Soc., 53, 305-324. https://dx.doi.org/10.1017/S0004972700017032

Zhao, X. Q. (2003). Dynamical Systems in Population Biology, CMS Books in Mathematics 16, Springer-Verlag, New York. http://dx.doi.org/10.1007/978-0-387-21761-1

\section{Copyrights}

Copyright for this article is retained by the author(s), with first publication rights granted to the journal.

This is an open-access article distributed under the terms and conditions of the Creative Commons Attribution license (http://creativecommons.org/licenses/by/4.0/). 